\title{
Corrigendum: Uncontrolled, Repetitive Eating of Small Amounts of Food or 'Grazing': Development and Evaluation of a New Measure of Atypical Eating
}

Brigitte Lane and Marianna Szabó

doi:10.1017/bec.2013.6, Published online by Cambridge University Press, 11 April 2013

Due to editing errors, the mean values for the Grazing Questionnaire in Table 2 were printed incorrectly, and a note explaining the derivation of DASS scores was omitted in Lane and Szabó (2013). Table 2 with the correct Grazing Questionnaire Mean values and a note regarding the DASS scores is below.

\section{TABLE 2}

Eating Patterns, Shape and Weight Concerns, Negative Emotions, and Eating Triggers in the Total Sample $(N=248)$, and in Women $(n=181)$ and Men $(n=67)$ Separately

\begin{tabular}{|c|c|c|c|c|c|c|c|c|}
\hline & \multicolumn{2}{|c|}{ Total sample } & \multicolumn{2}{|c|}{ Women } & \multicolumn{2}{|c|}{ Men } & \multirow[b]{2}{*}{$t$} & \multirow[b]{2}{*}{$d f$} \\
\hline & $\mathrm{M}$ & $S D$ & $\mathrm{M}$ & $S D$ & $\mathrm{M}$ & $S D$ & & \\
\hline Grazing (7 items) & 11.60 & 4.59 & 12.09 & 4.33 & 10.25 & 5.04 & $-2.84^{* *}$ & 245 \\
\hline Grazing (8 items) & 13.62 & 5.09 & 14.10 & 4.74 & 12.31 & 5.78 & $-2.48^{*}$ & 245 \\
\hline Binge eating (BES) & 10.68 & 6.82 & 12.04 & 6.94 & 7.14 & 5.03 & $-6.02^{* * *}$ & 161 \\
\hline Binge eating (BED) & 4.45 & 3.35 & 4.80 & 3.39 & 3.54 & 3.11 & $-2.76^{*}$ & 129 \\
\hline Chaotic eating & 21.62 & 5.09 & 8.52 & 8.02 & 6.70 & 5.76 & -1.70 & 244 \\
\hline Night eating & 13.26 & 5.31 & 13.64 & 5.51 & 12.24 & 4.62 & -1.85 & 246 \\
\hline Body Dissatisfaction & 15.18 & 9.54 & 17.74 & 9.3 & 8.39 & 6.34 & $-8.97^{* * *}$ & 174 \\
\hline Drive for Thinness & 8.41 & 7.45 & 10.36 & 7.36 & 3.14 & 4.60 & $-9.00^{* * *}$ & 180 \\
\hline Depression $^{a}$ & 9.68 & 8.61 & 10.01 & 8.49 & 8.55 & 8.90 & -1.25 & 244 \\
\hline Anxiety ${ }^{a}$ & 7.33 & 6.85 & 7.52 & 6.72 & 6.81 & 7.21 & -0.73 & 244 \\
\hline Stress ${ }^{a}$ & 13.47 & 8.80 & 14.49 & 8.45 & 10.72 & 9.20 & $-3.05^{* *}$ & 246 \\
\hline Restrained Eating & 26.28 & 9.59 & 28.23 & 9.12 & 20.40 & 8.57 & $-5.70^{* * *}$ & 227 \\
\hline Emotional Eating & 31.39 & 12.59 & 33.87 & 12.35 & 25.00 & 10.88 & $-5.11^{* * *}$ & 234 \\
\hline External Eating & 31.96 & 6.89 & 32.25 & 6.72 & 31.19 & 7.35 & -1.07 & 246 \\
\hline
\end{tabular}

Note: ${ }^{a}$ DASS-21 Depression, Anxiety and Stress scores have been doubled in accordance with guidelines (Lovibond \& Lovibond, 1995) to enable comparison with DASS-42 severity ratings. ${ }^{*} \mathrm{p}<.05,{ }^{* *} \mathrm{p}<.01,{ }^{* * *} \mathrm{p}<.001$, two-tailed.

Behaviour Change I Volume 31 I Number 3 I 2014 I pp. 222-223 I (C) The Author(s), published by Cambridge University Press on behalf of Australian Academic Press Pty Ltd 2014 I doi 10.1017/bec.2014.15 


\section{Reference}

Lane, B., \& Szabó, M. (2013). Uncontrolled, repetitive eating of small amounts of food or 'grazing': Development and evaluation of a new measure of atypical eating. Behaviour Change, 30, 57-73. doi:10.1017/bec.2013.6 